\title{
Ward identity and basis tensor gauge theory at one loop
}

\author{
Daniel J.H. Chung* \\ Department of Physics, University of Wisconsin-Madison, Madison, Wisconsin 53706, USA
}

(Received 30 January 2018; published 7 June 2018)

\begin{abstract}
Basis tensor gauge theory (BTGT) is a reformulation of ordinary gauge theory that is an analog of the vierbein formulation of gravity and is related to the Wilson line formulation. To match ordinary gauge theories coupled to matter, the BTGT formalism requires a continuous symmetry that we call the BTGT symmetry in addition to the ordinary gauge symmetry. After classically interpreting the BTGT symmetry, we construct using the BTGT formalism the Ward identities associated with the BTGT symmetry and the ordinary gauge symmetry. For a way of testing the quantum stability and the consistency of the Ward identities with a known regularization method, we explicitly renormalize the scalar QED at one loop using dimensional regularization using the BTGT formalism.
\end{abstract}

DOI: 10.1103/PhysRevD.97.125003

\section{INTRODUCTION}

Gauge theories (see, e.g., Refs. [1-8]) used to write the Standard Model of particle physics [8-17] are usually written in terms of fields that transform inhomogeneously under the gauge group; i.e., these are connections on principal bundles (see, e.g., Refs. [18,19]). In Ref. [20], we constructed a reformulation of ordinary gauge theories in analogy with the vierbein reformulation of general relativity. In particular, we constructed a replacement for the gauge field degree of freedom that transforms homogeneously under the $U(1)$ gauge group and satisfies certain constraints. Since the lowest-rank Lorentz tensor for such a field was shown to be 2 , the field $G^{\alpha}{ }_{\beta}$ (which replaces the usual $A_{\mu}$ gauge field) carries two Lorentz indices, transforms as a multiplicative $U(1)$ phase representation, and satisfies a nonlinear constraint equation.

This constraint equation was solved in Ref. [20] in terms of $N$ unconstrained scalars $\theta^{a}(x)$ ( $N$ is the number of spacetime dimensions) with the help of constant matrices $\left(H^{a}\right)^{\mu}{ }_{\nu}$, which in some sense are analogous to the Clifford algebra basis matrices in the well-known spinor field theory constructions. The field theory of $\theta^{a}(x)$ is what we called basis tensor gauge theory (BTGT). The closest cousin of the $\theta^{a}$ field is the Wilson line (e.g., Refs. [21-28] and references therein), but $\theta^{a}(x)$ has an appealing simplicity of being manifestly local.

\section{*danielchung@wisc.edu}

Published by the American Physical Society under the terms of the Creative Commons Attribution 4.0 International license. Further distribution of this work must maintain attribution to the author(s) and the published article's title, journal citation, and DOI. Funded by SCOAP .
To match the BTGTaction to that of the usual gauge theory coupled to scalars, we had to impose a new local symmetry in Ref. [20], which we will call the BTGT symmetry. In this paper, we investigate the Ward identities associated with the BTGT symmetry and the usual $U(1)$ gauge symmetry within the BTGT formalism as a step in building practical computational tools and checking the theory's quantum stability. First, we find that the BTGT symmetry current itself can be classically interpreted as a decomposition of $A^{\mu}$ equations of motion in basis tensor components. We will also find a relationship of a particular combination of BTGT current conservation and the residual gauge symmetry current conservation in the $\xi$-fixed ordinary $A^{\mu}$ field theory formalism. Next, we use the effective action formalism to derive the BTGT and the $U(1)$ Ward identities in both configuration space and momentum space within the BTGT formalism. These identities are then explicitly applied to one-loop renormalization of scalar QED. We find that dimensional regularization preserves the BTGT symmetry [in addition to the $U(1)$ as expected]. The explicit computations also highlight the utility of the basis tensor star product $J \star_{C} K \equiv$ $J^{\mu}\left(H^{c}\right)_{\mu \nu} K^{\nu}$ in computing the Feynman graphs within the BTGT formalism. Through this explicit renormalization exercise, we confirm that BTGT is stable at one loop.

The order of presentation is as follows. In the next section, we give a quick overview of the basis tensor gauge theory formalism. In Sec. III, we construct the BTGT Noether current and give a classical interpretation. In Sec. IV, we briefly review the effective action method of generating Ward identities. In Sec. V, we compute the Ward identities for both the BTGT symmetry and $U(1)$ symmetry in the context of the BTGT formalism. In Sec. VI, we use the BTGT formalism Feynman rules to explicitly renormalize the scalar QED at one loop, checking the quantum stability of the theory as well as the consistency of the dimensional 
regularization with the Ward identities. We conclude in Sec. VII by speculating on future research directions. The Appendix presents basic identities of $\left(H^{a}\right)^{\mu}{ }_{\nu}$ useful for Feynman diagram computations. There, we also point out a minor typo in Eq. (36) of Ref. [20].

\section{REVIEW OF BTGT FORMALISM}

In this section, we give a very brief review of Ref. [20]. For more details, we refer the reader to the original article.

A vierbein formulation of Einstein gravity relies on finding a basis of spacetime vector fields that transforms as $(1,1)$ under $S O(3,1) \otimes$ diffeomorphism. By contracting with vierbeins, a nonsinglet diffeomorphism tensor turns into a set of diffeomorphism scalar fields that transform as nonsinglet $S O(3,1)$ tensor components. The vierbein's relationship with the Christoffel symbols (i.e., the gravitational gauge fields) can be viewed as the vierbeins being solutions to a set of nonlinear partial differential equations involving the Christoffel symbols. The vierbein analog in the context in which ordinary compact Lie groups replace diffeomorphism group is the focus of BTGT.

In the $U(1)$ BTGT of Ref. [20], the field $G^{\alpha}{ }_{\beta}$ is the analog of the gravitational vierbein, and it transforms as $(2,1)$ under $S O(3,1) \otimes U(1)$. The constraint of matching to the usual $U(1)$ connection (i.e., the analog of the gravitational vierbein relationship to the Christoffel symbols $\Gamma_{\mu \nu}^{\alpha}$ ) is

$$
A_{\mu}=-i\left(G^{-1}\right)_{\beta}^{\alpha} \partial_{\alpha} G^{\beta}{ }_{\mu} .
$$

The number of degrees of freedom in $G^{\alpha}{ }_{\beta}$ is reduced by introducing BTGT fields $\theta^{a}$ (i.e., four scalar fields in four spacetime dimensions) through

$$
G^{\beta}{ }_{\mu}(x)=\left(e^{i \theta^{a}(x) H^{a}}\right)_{\mu}^{\beta},
$$

where the constant matrices $H^{a}$ satisfy $\left[H^{a}, H^{b}\right]=0$ and $H^{a}$ transforms like a Rank-2 tensor under Lorentz transformations. ${ }^{1}$ An explicit representation of $H^{a}$ is discussed in the Appendix. The relationship of $\theta^{a}$ to $A_{\mu}$ is then

$$
A_{\mu}(x)=\sum_{a}\left(H^{a}\right)_{\mu}^{\alpha} \partial_{\alpha} \theta^{a},
$$

which says that $\theta^{a}$ is similar to the Wilson line.

To construct a field theory of $\theta^{a}$ (i.e., BTGT theory) that matches the ordinary gauge theory, one must impose the following independent continuous symmetries:

(1) Ordinary gauge symmetry: If $\phi$ has charge $e$ under $U(1)$, then the $U(1)$ gauge symmetry is the set $\left\{\delta \phi=i e \theta \phi, \delta \phi^{*}=-i e \theta \phi^{*}, \delta \theta^{a}=\theta\right\}$.

(2) BTGT symmetry: Vary just the BTGT field by a restricted class of functions $\delta \theta^{a}=Z_{\sim}^{a}(x)$, where $\left(H^{a}\right)^{\alpha}{ }_{\mu} \partial_{\alpha} Z_{\sim}^{a}(x)=0$, and there is no sum over the repeated $a$ index in this equation.

\footnotetext{
${ }^{1}$ This is analogous to normalizing the gravitational vierbein dot products to reduce to the Minkowski metric.
}

At the renormalizable level, this set of symmetries reproduces the ordinary $U(1)$ gauge theory action. For example, in the case of scalar QED, the partition function is

$$
\mathscr{L}=\int D \theta^{a} D \phi D \phi^{*} e^{i S\left[\theta^{a}, \phi, \phi^{*}\right]},
$$

where the Lagrangian terms for the $\xi$-gauge fixed action are given by Eqs. (14)-(17).

Correlators of certain differences of $\theta^{a}$ map to correlators of integrals over $A_{\mu}$.

\section{CLASSICAL BTGT SYMMETRY CURRENT}

To write scalar QED in the BTGT formalism, a new local symmetry [in addition to the usual $U(1)$ gauge symmetry] was introduced in Ref. [20], which we will refer to as the BTGT symmetry in this paper. To have a physical interpretation of the continuous BTGT symmetry, we construct the Noether current associated with this symmetry in this section. More explicitly, we seek the Noether currents associated with the BTGT symmetry defined as

$$
\delta \theta^{a}={\underset{\sim}{Z}}^{a}(x)
$$

satisfying the constraint equation

$$
\left(H^{a}\right)^{\alpha}{ }_{\mu} \partial_{\alpha} Z_{\sim}^{a}(x)=0,
$$

where there is no sum over the repeated $a$ index in this equation. Note that, even though $Z_{\sim}^{a}(x)$ is reminiscent of a pure $U(1)$ gauge field function $\theta(x)$ appearing in $\delta A_{\mu}=\partial_{\mu} \theta$, there is no gauge charged tensor transformation here since all the gauge charged matter fields are held fixed and only the $\theta^{a}$ transforms as $\delta \theta^{a}=Z_{\sim}^{a}(x)$. Furthermore, each $Z^{a}(x)$ for different $a$ indices are independent.

To gain some intuition regarding the mathematical procedure for constructing the Noether current of a constrained symmetry representation, it is useful to review a mathematically analogous more familiar symmetry: the residual gauge transformation of a $\xi$-gauge fixed scalar QED theory. With the gauge-fixing term

$$
\mathcal{L}_{\mathrm{GF}}=\frac{-1}{2 \xi}(\partial A)^{2},
$$

there still exists a continuous residual gauge transformation,

$$
\delta A_{\mu}=\partial_{\mu} h, \quad \delta \phi=i e h \phi, \ldots,
$$

where

$$
\square h=0 .
$$

Equation (9) is the analog of the constraint equation (6). Using the standard Noether construction with the 
nonsymmetry deformation parametrized through an arbitrary continuous function $\varepsilon(x) \mathrm{as}^{2}$

$$
\delta A_{\mu}=\partial_{\mu}[\varepsilon(x) h(x)],
$$

it is straightforward to show

$$
\partial_{\mu} j_{R}^{\mu}=0
$$

where

$$
j_{R}^{\mu} \equiv \partial^{\mu}\left(\partial_{\nu} A^{\nu}\right)
$$

Since the classical equations of motion in the $\xi$ gauge satisfies

$$
\partial_{\nu}\left[\partial_{\mu} F^{\mu \nu}+\frac{1}{\xi} \partial^{\nu}\left(\partial_{\mu} A^{\mu}\right)-J_{U(1)}^{\nu}\right]=0,
$$

where $J_{U(1)}^{\nu}$ is a Noether current associated with the global subgroup of $U(1)$, we see that Eq. (11) indeed is satisfied, owing to the antisymmetric property of the field strength tensor and the global $U(1)$ current conservation.

We can carry out the same exercise with the BTGT symmetry of Eq. (5). We consider the $\xi$-gauge fixed scalar QED in the BTGT basis [20]:

$$
\begin{gathered}
\mathcal{L}_{k 2}=\frac{-Z_{3}}{2} \partial_{\mu}\left(\partial_{\alpha} \theta^{a}\left(H^{a}\right)^{\alpha}{ }_{, \nu}\right) \partial^{\mu}\left(\partial_{\beta} \theta^{b}\left(H^{b}\right)^{\beta \nu}\right) \\
+\frac{Z_{3}}{2} \partial_{\mu}\left(\partial_{\alpha} \theta^{a}\left(H^{a}\right)^{\alpha}{ }_{, \nu}\right) \partial^{\nu}\left(\partial_{\beta} \theta^{b}\left(H^{b}\right)^{\beta \mu}\right), \\
\mathcal{L}_{I}=Z_{1}\left[i e \phi^{*} \partial^{\mu} \phi \partial_{\alpha} \theta^{a}\left(H^{a}\right)^{\alpha}{ }_{\mu}+\text { H.c. }\right] \\
+Z_{4} e^{2}|\phi|^{2} \partial_{\alpha} \theta^{a}\left(H^{a}\right)^{\alpha}{ }_{\mu} \partial_{\beta} \theta^{b}\left(H^{b}\right)^{\beta \mu}, \\
\mathcal{L}_{k s}=Z_{2}|\partial \phi|^{2}-m^{2} Z_{m}|\phi|^{2} \\
\mathcal{L}_{\mathrm{GF}} \equiv \frac{-1}{2 \xi}\left[\partial^{\mu} \partial_{\alpha} \theta^{a}\left(H^{a}\right)_{\mu}^{\alpha}\right]^{2} .
\end{gathered}
$$

In these expressions, the summation over repeated indices is implied, and we will assume below that repeated indices are summed unless stated otherwise explicitly or if it is clear from the context. This action is invariant under the BTGT symmetry equation (5). The gauge-fixing term $\mathcal{L}_{\mathrm{GF}}$ breaks the usual $U(1)$ gauge transformations, which when written in the BTGT formalism are

$$
\delta \phi=i e \theta \phi, \quad \delta \phi^{*}=-i e \theta \phi^{*}, \quad \delta \theta^{a}=\theta,
$$

\footnotetext{
${ }^{2}$ In principle, we can separate the functional variations more carefully since some $\varepsilon(x)$ choices will keep $\varepsilon h$ harmonic. Owing partly to Lorentz invariance, one can check that this does not change the results for the construction of local currents.
}

where $\theta$ is an arbitrary smooth function, but it does preserve the global $U(1)$ subgroup.

We find the conservation of the Noether current associated with the BTGT symmetry to be

$$
\partial_{\mu} \mathcal{B}_{a}^{\mu}=0
$$

where

$\mathcal{B}_{a}^{\mu}=\left(H^{a}\right)_{\lambda}^{\mu}\left[\partial^{\lambda} \sum_{b} \partial \star_{b} \partial \theta^{b}\left(1-\frac{1}{\xi}\right)-\partial^{\lambda} \square \theta^{a}+\mathcal{C}_{U(1) a}^{\lambda}(x)\right]$

$$
\mathcal{C}_{U(1) a}^{\mu} \equiv-\left[i e \phi^{*} \partial^{\mu} \phi+\text { H.c. }\right]-2\left[e^{2}|\phi|^{2} \partial^{\mu} \theta^{a}\right],
$$

where we have introduced the notation

$$
A \star_{b} B \equiv A^{\mu} B^{\nu}\left(H^{b}\right)_{\mu \nu} .
$$

This current is ordinary $U(1)$ gauge invariant if $\xi \rightarrow \infty$ since under Eq. (18) the current variation is

$$
\begin{aligned}
\delta \mathcal{B}_{a}^{\mu}= & \left(H^{a}\right)^{\mu}{ }_{\lambda}\left[\left(1-\frac{1}{\xi}\right) \partial^{\lambda}\left(\sum_{b} \partial{ }_{\star_{b}} \partial\right) \theta-\partial^{\lambda} \square \theta\right. \\
& -\left[i e \phi^{*} \partial^{\mu}(i e \theta \phi)-i e \phi \partial^{\mu}\left(-i e \theta \phi^{*}\right)\right] \\
& \left.-2 e^{2}|\phi|^{2} \partial^{\lambda} \theta\right] \\
= & \frac{-1}{\xi}\left(H^{a}\right)^{\mu}{ }_{\lambda} \partial^{\lambda} \square \theta .
\end{aligned}
$$

From this, one also sees that the current is invariant under residual gauge transformations of Eq. (8) even if $\xi \neq \infty$. Because of the $\left(H^{a}\right)_{\lambda}^{\mu}$ in front of Eq. (20), it is also invariant under the BTGT symmetry of Eq. (5).

The BTGT current equation (20) can be interpreted in terms of $A_{\mu}$ by noting that the classical relationship

$$
A_{\mu}=\sum_{b}\left(H^{b}\right)^{\alpha}{ }_{\mu} \partial_{\alpha} \theta^{b}
$$

(see Ref. [20] for details) allows us to identify

$$
\sum_{a} \mathcal{B}_{a}^{\mu}=\partial^{\mu} \partial \cdot A\left(1-\frac{1}{\xi}\right)-\square A^{\mu}+J_{U(1)}^{\mu},
$$

where

$$
J_{U(1)}^{\mu} \equiv-\left[i e \phi^{*} \partial^{\mu} \phi+\text { H.c. }\right]-2 e^{2}|\phi|^{2} A^{\mu} .
$$

Hence, the sum of the BTGT currents itself is the equation of motion in terms of the usual gauge field $A_{\mu}$. As we will see below, the equation of motion for $\theta^{a}$ differs from the 
equation of motion for $A_{\mu}$ by a derivative. Hence, the BTGT current can be interpreted as the decomposition of the $A^{\mu}$ equation of motion in basis tensor components (to be distinguished from the decomposition of the $\theta^{a}$ equation of motion in basis tensor components).

To check $\mathcal{B}_{a}^{\mu}$ conservation using the classical equations of motion, note Eq. (19) can be rewritten as

$\partial \star_{a}\left[\partial \sum_{b} \partial \star_{b} \partial \theta^{b}\left(1-\frac{1}{\xi}\right)-\partial \square \theta^{a}+\mathcal{C}_{U(1) a}(x)\right]=0$

for each $a$ choice. Since this is precisely the equation of motion of $\theta^{a}$, the BTGT classical current is manifestly conserved for classical $\theta^{b}$ fields satisfying the equation of motion. On the other hand, the equation of motion for $A^{\mu}$ is the sum of the currents shown in Eq. (26) and not the equation of motion for $\theta^{b}$ itself; i.e., the difference is a particular type of derivative.

Now, let us combine

$$
\partial_{\mu} \sum_{a} \mathcal{B}_{a}^{\mu}=0
$$

with the Noether current conservation coming from the global subgroup of the $U(1)$ gauge symmetry (which obviously is not broken even for finite $\xi$ ):

$$
\partial_{\mu} J_{U(1)}^{\mu}=0 .
$$

This means that the ordinary $U(1)$ current conservation and the sum of the BTGT current conservation together enforce

$$
\frac{1}{\xi} \square \partial \cdot A=0,
$$

which is the same as Eq. (11) enforced by the residual gauge symmetry.

\section{BRIEF REVIEW OF EFFECTIVE ACTION GENERATING WARD IDENTITIES}

We would now like to study the quantum current conservation associated with the BTGT symmetry, i.e., generate Ward identities. For this goal, we briefly review here the effective action formalism for generating Ward identities [29]. This allows us to then reduce the generation of Ward identities to a set of functional derivatives, which we will use to obtain explicit Ward identities for the BTGT and gauge symmetry.

Let $\varphi$ be a vector of fields [e.g., $\varphi=\left(\theta^{a}, \phi, \phi^{*}, \ldots\right)$ and $\left.J=\left(J_{a}, J_{\phi}, J_{\phi^{*}}, \ldots\right)\right]$. The generating functional $W$ for the connected Green's functions $\mathcal{G}_{c}^{(n)}$ is

$$
\begin{gathered}
i W[J] \equiv \ln Z[J] \\
=\sum_{n=0}^{\infty} \frac{i^{n}}{n !} \int d^{4} x_{1} \ldots d^{4} x_{n} J\left(x_{1}\right) \ldots J\left(x_{n}\right) \mathcal{G}_{c}^{(n)}\left(x_{1}, \ldots, x_{n}\right),
\end{gathered}
$$

and the effective action is its Legendre transform

$$
\Gamma[\bar{\varphi}] \equiv W[J(\bar{\varphi})]-\int d^{4} x J(\bar{\varphi}) \bar{\varphi},
$$

where

$$
\bar{\varphi}(x) \equiv \frac{\delta W[J]}{\delta J(x)} .
$$

The effective action $\Gamma$ can be interpreted as a collection of amplitudes

$\Gamma\left[\varphi_{c}\right]=\sum_{n} \frac{1}{n !} \int d^{4} x_{1} \ldots d^{4} x_{n} \varphi_{c}\left(x_{1}\right) \ldots \varphi_{c}\left(x_{n}\right) \Gamma^{(n)}\left(x_{1}, \ldots, x_{n}\right)$,

where $\Gamma^{(n)}\left(x_{1}, \ldots, x_{n}\right)$ are external momenta truncated (with full propagators) 1PI graphs.

It is well known that one can choose counter terms perturbatively such that only gauge-invariant counterterms renormalize the theory as long as the regulators do not spoil gauge invariance. This leads to the gauge-invariant effective action if we subtract out the tree-level gauge-fixing action from the total effective action:

$$
\hat{\Gamma}\left[\varphi_{c}\right] \equiv \Gamma-S_{\mathrm{GF}}
$$

At this point, one simply applies the functional derivative representation of the symmetry transformation and picks out functionally independent coefficients to generate the Ward identities.

As a simple illustration, consider scalar QED in the usual $A_{\mu}$ gauge field representation. The gauge transformation

$$
\delta \phi=i e \theta \phi, \quad \delta \phi^{*}=-i e \theta \phi^{*}, \quad \delta A_{\mu}=\partial_{\mu} \theta
$$

mixes the three-point function involving $\left(A_{\mu}, \phi^{*}, \phi\right)$ fields and two-point functions obtained from removing $A_{\mu}$ due to the inhomogeneous nature of $\delta A_{\mu}$. Hence, the relevant components of the effective action are

$$
\begin{aligned}
\hat{\Gamma} \ni I \equiv & \int d^{4} x d^{4} y d^{4} z \phi^{*}(x) \phi(y) A^{\mu}(z) \hat{\Gamma}_{\mu}^{(1,1,1)}(x, y, z) \\
& +\int d^{4} x d^{4} y \phi^{*}(x) \phi(y) \hat{\Gamma}^{(1,1,0)}(x, y) .
\end{aligned}
$$

To apply the symmetry transformations on this object, one needs a functional representation of Eq. (38),

$$
\mathcal{O}_{0}\left(\phi^{*}, \phi, A^{\nu}\right)=\left(-i e \theta \phi^{*}, i e \theta \phi, \partial^{\nu} \theta\right),
$$

where 


$$
\begin{aligned}
\mathcal{O}_{0} \equiv & \int d^{4} y^{\prime} \partial^{\nu} \theta\left(y^{\prime}\right) \frac{\delta}{\delta A^{\nu}\left(y^{\prime}\right)}+i e \int d^{4} y^{\prime} \theta\left(y^{\prime}\right) \phi\left(y^{\prime}\right) \frac{\delta}{\delta \phi\left(y^{\prime}\right)} \\
& -i e \int d^{4} y^{\prime} \theta\left(y^{\prime}\right) \phi^{*}\left(y^{\prime}\right) \frac{\delta}{\delta \phi^{*}\left(y^{\prime}\right)} .
\end{aligned}
$$

Ward identities are generated through

$$
\left.\frac{\delta}{\delta \theta\left(z_{3}\right)} \frac{\delta}{\delta \phi^{*}\left(z_{2}\right)} \frac{\delta}{\delta \phi\left(z_{1}\right)} \mathcal{O}_{0} I\right|_{A=0}=0
$$

Explicitly, one finds the well-known Ward identity:

$$
\begin{aligned}
\partial^{\mu} \hat{\Gamma}_{\mu}^{(1,1,1)}\left(z_{2}, z_{1}, z_{3}\right)= & i e \delta^{(4)}\left(z_{1}-z_{3}\right) \hat{\Gamma}^{(1,1,0)}\left(z_{2}, z_{1}\right) \\
& -i e \delta^{(4)}\left(z_{2}-z_{3}\right) \hat{\Gamma}^{(1,1,0)}\left(z_{2}, z_{1}\right) .
\end{aligned}
$$

\section{BTGT FORMALISM WARD IDENTITIES}

In this section, we use the method of the previous section to derive the Ward identities in the BTGT formalism both for the $U(1)$ gauge symmetry and the BTGT symmetry.

\section{A. $U(1)$ in BTGT formalism}

Here, we consider the $U(1)$ gauge symmetry transformations of Eq. (18). The functional derivative representation analog of Eq. (41) for this symmetry is

$$
\begin{aligned}
\mathcal{O}_{U(1)} \equiv & \sum_{f} \int d^{4} y^{\prime} \theta\left(y^{\prime}\right) \frac{\delta}{\delta \theta^{f}\left(y^{\prime}\right)} \\
& +i e\left[\int d^{4} y^{\prime} \theta\left(y^{\prime}\right) \phi\left(y^{\prime}\right) \frac{\delta}{\delta \phi\left(y^{\prime}\right)}\right. \\
& \left.-\int d^{4} y^{\prime} \theta\left(y^{\prime}\right) \phi^{*}\left(y^{\prime}\right) \frac{\delta}{\delta \phi^{*}\left(y^{\prime}\right)}\right] .
\end{aligned}
$$

First, applying this operator to the two-point function

$$
\hat{\Gamma} \ni \frac{1}{2} \int d^{4} x d^{4} y \sum_{a b} \hat{\Gamma}_{a b}^{(0,0,2)}(x, y) \theta^{a}(x) \theta^{b}(y),
$$

we find

$$
\begin{aligned}
I_{2} \equiv & \sum_{a b} \int d^{4} x \int d^{4} y \hat{\Gamma}_{a b}^{(0,0,2)}(x, y) \theta(x) \theta^{b}(y) \\
& +\sum_{a b} \int d^{4} y \int d^{4} x \hat{\Gamma}_{a b}^{(0,0,2)}(x, y) \theta^{a}(x) \theta(y)=0 .
\end{aligned}
$$

Next, we apply the functional independence constraint to obtain

$$
\begin{aligned}
& \frac{\delta}{\delta \theta\left(z_{1}\right)} \frac{\delta}{\delta \theta^{f}\left(z_{2}\right)} I_{2} \\
& =\sum_{a}\left(\hat{\Gamma}_{a f}^{(0,0,2)}\left(z_{1}, z_{2}\right)+\int d^{4} x \hat{\Gamma}_{f a}^{(0,0,2)}\left(z_{2}, z_{1}\right)\right)=0 .
\end{aligned}
$$

Since the two-point function is symmetric in the af symbol, we conclude

$$
\sum_{a} \hat{\Gamma}_{a b}^{(0,0,2)}(z, x)=0
$$

and

$$
\sum_{b} \hat{\Gamma}_{a b}^{(0,0,2)}(x, z)=0
$$

(Since we have been a bit overexplicit here, we will henceforth omit writing redundant combinations derivable from the index/variable permutations.)

Let us see if this is satisfied by the tree-level propagator. Note that in Fourier space the two-point coefficient is given by the inverse of the propagator,

$$
\Gamma_{f c}^{(0,0,2)}(k)=i\left(\Delta^{-1}\right)_{f c},
$$

where $\Delta_{b c}(k)$ is the propagator defined by

$$
\left\langle\theta^{b}(y) \theta^{c}(z)\right\rangle=\int \frac{d^{4} l}{(2 \pi)^{4}} e^{-i l \cdot(y-z)} \Delta_{b c}(l) .
$$

To obtain $\hat{\Gamma}_{a b}$, we subtract out the gauge-fixing term:

$$
S_{\mathrm{GF}}=\frac{-1}{2 \xi} \int d^{4} x \theta^{a}\left(H^{a}\right)_{\mu}^{\alpha}\left(H^{b}\right)_{\beta}^{\lambda} \partial^{\mu} \partial_{\alpha} \partial^{\beta} \partial_{\lambda} \theta^{b} .
$$

We can rewrite this as

$$
S_{\mathrm{GF}}=\frac{1}{2 !} \int d^{4} x d^{4} z \Gamma_{a b}^{\mathrm{GF}}(x, z) \theta^{a}(x) \theta^{b}(z)
$$

and Fourier transform as

$$
\Gamma_{a b}^{\mathrm{GF}}(x, z) \equiv \int \frac{d^{4} k}{(2 \pi)^{4}} e^{i k \cdot(x-z)} \Gamma_{a b}^{\mathrm{GF}}(k)
$$

to obtain

$$
\Gamma_{a b}^{\mathrm{GF}}(k)=\frac{-1}{\xi}\left(H^{a}\right)^{\alpha}{ }_{\mu}\left(H^{b}\right)^{\lambda}{ }_{\beta} k^{\mu} k_{\alpha} k^{\beta} k_{\lambda} .
$$

The gauge-fixing term subtraction therefore gives

$$
\hat{\Gamma}_{f c}^{(0,0,2)}(k)=\Gamma_{f c}^{(0,0,2)}(k)+\frac{1}{\xi}\left(k \star_{f} k\right)\left(k \star_{c} k\right),
$$


and the Ward identity is

$$
\sum_{f}\left[i\left(\Delta^{-1}\right)_{f c}+\frac{1}{\xi}\left(k \star_{f} k\right)\left(k \star_{c} k\right)\right]=0
$$

to all orders in perturbation theory.

At tree level, we have after subtraction the expression

$$
\hat{\Gamma}_{f c}^{(0,0,2)}(k)=-k^{2} \delta^{f}{ }_{c} k \star_{f} k+\left(k \star_{f} k\right)\left(k \star_{c} k\right) .
$$

We can sum over $f$ and find

$$
\sum_{f} \hat{\Gamma}_{f c}^{(0,0,2)}(k)=-k^{2} k \star_{c} k+k^{2}\left(k \star_{c} k\right)=0,
$$

confirming the Ward identity for $U(1)$ at tree level.

Next, let us consider the three-point function. Operating $\mathcal{O}_{U(1)}$ on

$$
\begin{aligned}
\hat{\Gamma} \ni & \sum_{c} \int d^{4} x d^{4} y d^{4} z \phi^{*}(x) \phi(y) \theta^{c}(z) \hat{\Gamma}_{c}^{(1,1,1)}(x, y, z) \\
& +\int d^{4} x d^{4} y \phi^{*}(x) \phi(y) \hat{\Gamma}^{(1,1,0)}(x, y),
\end{aligned}
$$

we find for the three-point function Ward identity

$$
\begin{aligned}
-\sum_{c} \hat{\Gamma}_{c}^{(1,1,1)}\left(z_{2}, z_{1}, z_{3}\right)= & i e \delta^{(4)}\left(z_{1}-z_{3}\right) \hat{\Gamma}^{(1,1,0)}\left(z_{2}, z_{1}\right) \\
& -i e \delta^{(4)}\left(z_{2}-z_{3}\right) \hat{\Gamma}^{(1,1,0)}\left(z_{2}, z_{1}\right) .
\end{aligned}
$$

In Fourier space, with Feynman diagrams drawn with outgoing scalar momentum $p_{2}=p$ and incoming scalar momentum $p_{1}=-q$, and incoming $\theta^{c}$ field momentum $p_{3}=-k$, we find

$$
-\sum_{c} \hat{G}_{c}^{(1,1,1)}(p,-q,-k)=i e\left[\hat{G}^{(1,1,0)}(p,-q-k)-\hat{G}^{(1,1,0)}(p-k,-q)\right] \text {, }
$$

where $\hat{G}_{c}$ is the Fourier space representation of $\hat{\Gamma}_{c}$, e.g., $\hat{\Gamma}_{c}^{(1,1,1)}\left(z_{2}, z_{1}, z_{3}\right)=\sum_{c} \int \prod_{n=1}^{3} \frac{d^{4} k_{n}}{(2 \pi)^{4}} e^{i k_{n} \cdot z_{n}} \hat{G}_{c}^{(1,1,1)}\left(k_{2}, k_{1}, k_{3}\right)$.

At tree level, Eq. (62) is

$$
\begin{aligned}
-\sum_{c} e(q+p) \star_{c} k= & (i e) i[i p \cdot(i[-q-k]) \\
& -i(p-k) \cdot(i[-q])] \\
= & -e(p+q) \cdot k,
\end{aligned}
$$

which indeed is an identity.

\section{B. BTGT symmetry}

Next, we investigate the Ward identities associated with the BTGT symmetry (5). The functional derivative representation is

$$
\delta \theta^{a} \equiv \mathcal{O}_{B}^{(a)} \theta^{a}
$$

where

$$
\mathcal{O}_{B}^{(a)} \equiv \int d^{4} z Z_{\sim}^{a}(z) \frac{\delta}{\delta \theta^{a}(z)} \text { no sum over } a
$$

Apply this to the two-point function

$$
\Gamma \ni \frac{1}{2} \sum_{a b} \int d^{4} x d^{4} y \hat{\Gamma}_{a b}^{(0,0,2)}(x, y) \theta^{a}(x) \theta^{b}(y),
$$

which is symmetric in both $a \leftrightarrow b$ and $x \leftrightarrow y$. We find

$$
\sum_{c} \int d^{4} x \hat{\Gamma}_{c b}^{(0,0,2)}(x, y) \underset{\sim}{Z^{c}}(x)=0
$$

We cannot take a simple functional derivative with respect to $Z^{c}(x)$ because that variable is constrained. One can solve the constraint trivially in Fourier space,

$$
{\underset{\sim}{Z}}^{a}(x)=\int \frac{d^{4} k}{(2 \pi)^{4}} e^{i \sum_{b \neq a} k \star_{b} x} F^{a}(k),
$$

where $F^{a}(k)$ is unconstrained. Equation (69) implies that the two-point contribution in momentum space satisfies

$$
\hat{\Gamma}_{c b}\left(K_{\beta}^{(\perp c)}\right)=0 \text {, }
$$

where 


$$
K_{\beta}^{(\perp c)}(k) \equiv \sum_{b \neq c} k_{\alpha}\left(H^{b}\right)_{\beta}^{\alpha}
$$

is the momentum vector with the $\left(H^{c}\right)^{\alpha}{ }_{\beta}$ component projected out.

This can be trivially checked with the tree-level action

$$
\hat{\Gamma}_{f c}(q)=-q^{2} \delta^{f c} q \star_{f} q+\left(q \star_{f} q\right)\left(q \star_{c} q\right),
$$

where there is no sum over the repeated indices here. Since

$$
K^{(\perp c)}(k) \cdot K^{(\perp c)}(k)=\sum_{m \neq c} k \star_{m} k
$$

and

$$
K^{(\perp c)}(k) \star_{c} K^{(\perp c)}(k)=0,
$$

we see that Eq. (71) is satisfied at the tree level.

At the three-point function level, the relevant terms in the effective action are

$$
\begin{aligned}
\hat{\Gamma} \ni & \sum_{c} \int d^{4} x d^{4} y d^{4} z \phi^{*}(x) \phi(y) \theta^{c}(z) \hat{\Gamma}_{c}^{(1,1,1)}(x, y, z) \\
& +\int d^{4} x d^{4} y \phi^{*}(x) \phi(y) \hat{\Gamma}^{(1,1,0)}(x, y) .
\end{aligned}
$$

Acting on this with $\mathcal{O}_{B}^{(a)}$, imposing BTGT symmetry, and taking functional derivatives with respect to unconstrained variables, we find

$$
\int d^{4} z Z_{\sim}^{a}(z) \hat{\Gamma}_{a}^{(1,1,1)}(x, y, z)=0 \text {. }
$$

As in Eq. (71), we can simplify this expression further in Fourier space where the constraint on $Z_{\sim}^{a}(z)$ can be trivially solved. Defining

$\hat{\Gamma}_{a}^{(1,1,1)}\left(x_{1}, x_{2}, x_{3}\right)=\int \prod_{n=1}^{3} \frac{d^{4} k_{n}}{(2 \pi)^{4}} e^{i \sum_{r=1}^{3} k_{r} \cdot x_{r}} \hat{G}_{a}^{(1,1,1)}\left(k_{1}, k_{2}, k_{3}\right)$,

Eq. (77) becomes

$$
\hat{G}_{a}^{(1,1,1)}\left(k_{1}^{\mu}, k_{2}^{\mu},-\left[K^{(\perp a)}(k)\right]^{\mu}\right)=0 \text {. }
$$

Since

$$
\hat{G}_{a}^{(1,1,1)}\left(k_{1}, k_{2}, k_{3}\right) \propto-e\left(k_{1}-k_{2}\right) \star_{a} k_{3},
$$

we see that Eq. (79) is manifestly satisfied at tree level, owing to the orthogonality property manifest in Eq. (A3).

\section{ONE-LOOP RENORMALIZATION}

One way to check the quantum stability of the BTGT formalism is to explicitly renormalize at one loop. Hence, in this section, we write down the Feynman rules for the scalar QED specified by Eqs. (14)-(17) in the BTGT formalism and explicitly renormalize at one loop. We will employ dimensional regularization and check that it is consistent with the BTGT Ward identity [and the $U(1)$ Ward identity]. The minimal subtraction renormalization scheme is used whenever we want to compare the renormalization constants $Z_{i}$ with each other. Some of the useful identities for the computation are given in the Appendix.

\section{A. Action and Feynman rules}

Here, we will write down the Feynman rules for scalar QED in the BTGT formulation specified by Eqs. (14)-(17). We will use the summation convention that we sum over all repeated latin indices unless specified otherwise. For our objective of testing the BTGT coupling at one loop, we have set the scalar quartic self-coupling to zero at tree level. The Feynman rules for this scalar QED theory are shown in Fig. 1. In the figure, the photon propagator has a factor written in terms of

$$
\left(K_{(b)}\right)_{\mu} \equiv\left(H^{b}\right)^{\psi}{ }_{\mu} \frac{k_{\psi}}{k_{\alpha} k_{\beta}\left(H^{b}\right)^{\alpha \beta}}=\left(H^{b}\right)^{\psi}{ }_{\mu} \frac{k_{\psi}}{k_{\star_{b}} k} .
$$

In the rest of this section, we use these Feynman rules in the Feynman gauge $(\xi=1)$ for an explicit one-loop renormalization of scalar QED.

\section{B. Vacuum polarization}

In this subsection, we compute the two vacuum polarization graphs shown in Fig. 2. All diagrams in this subsection and the subsequent subsections will be computed in the Feynman gauge.
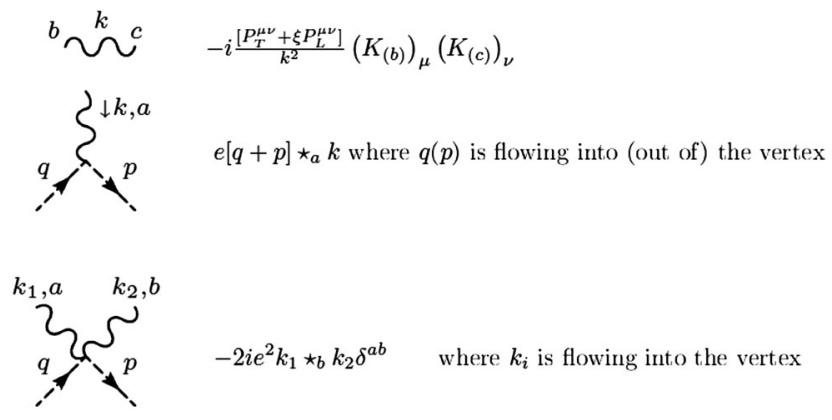

${ }^{a} \simeq \sim \sim^{b} \quad-i\left(Z_{3}-1\right) k^{2} k_{\alpha} k_{\beta}\left(H^{a}\right)^{\alpha}{ }_{\nu}\left(H^{b}\right)^{\beta}{ }_{\mu} P_{T}^{\mu \nu}$

FIG. 1. Feynman rules for the BTGT theory. The counterterm for the $\left\langle\theta^{a} \theta^{b}\right\rangle$ is shown explicitly to emphasize that only gaugeinvariant counterterms are needed just as in the usual connection formalism. 


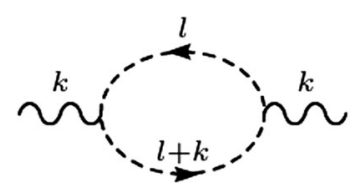

(a)

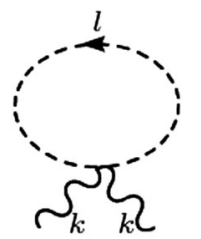

(b)
FIG. 2. Vacuum polarization diagrams are shown in (a)-(b).

The first graph we consider is the one of Fig. 2(a). The Feynman rules give

$$
\begin{aligned}
i \Pi_{1}^{a b}= & \int \frac{d^{4} l}{(2 \pi)^{4}} e(2 l+k) \star_{a} k\left(\frac{i}{l^{2}-m^{2}}\right)\left[-e(2 l+k) \star_{b} k\right] \\
& \times\left(\frac{i}{(k+l)^{2}-m^{2}}\right) .
\end{aligned}
$$

The structure of this diagram is similar to that of ordinary scalar QED other than the appearance of star products in the numerator,

$$
i \Pi_{1}^{a b}=k_{\alpha}\left(H^{a}\right)^{\alpha \mu} k_{\beta}\left(H^{b}\right)^{\beta \nu}\left(I_{1}^{\text {ordinary }}\right)_{\mu \nu},
$$

where

$$
\begin{aligned}
\left(I_{1}^{\text {ordinary }}\right)_{\mu \nu} \equiv & 2 e^{2}\left[g_{\mu \nu}\left(-\frac{1}{6} k^{2}+m^{2}\right)+\frac{1}{6} k_{\mu} k_{\nu}\right] \frac{i}{(4 \pi)^{2}} \\
& \times\left(\frac{2}{\varepsilon}+\cdots\right) .
\end{aligned}
$$

The quartic vertex graph of Fig. 2(b) gives

$$
\begin{aligned}
i \Pi_{2}^{a b} & =(-1)\left(-2 i e^{2} k \star_{b} k \delta^{a b}\right) \int \frac{d^{4} l}{(2 \pi)^{4}} \frac{i}{l^{2}-m^{2}} \\
& =k \star_{b} k \delta^{a b} I_{2}^{\text {ordinary }},
\end{aligned}
$$

where

$$
I_{2}^{\text {ordinary }}=-\left(2 e^{2}\right) \frac{i}{(4 \pi)^{2}} \frac{2}{\varepsilon} m^{2}+\cdots
$$

We can add Eqs. (83) and (86) to obtain

$$
\begin{aligned}
i \Pi_{1}^{a b}(k)+i \Pi_{2}^{a b}(k)= & +k_{\alpha}\left(H^{a}\right)^{\alpha \mu} k_{\beta}\left(H^{b}\right)^{\beta \nu} 2 e^{2}\left(-\frac{1}{6} k^{2}\right) \\
& \times P_{T \mu \nu} \frac{i}{(4 \pi)^{2}}\left(\frac{2}{\varepsilon}+\cdots\right) .
\end{aligned}
$$

Note that the scalar mass-dependent longitudinal mode has decoupled from the photon propagator, restoring gauge invariance (i.e., leaving the term proportional to the transverse projection operator $P_{T \mu \nu}$ ). Equation (88) generates the counterterm

$$
Z_{3}=1-\frac{e^{2}}{3} \frac{1}{(4 \pi)^{2}} \frac{2}{\varepsilon}
$$

for example, in the minimal subtraction scheme.

Let us explicitly check that the Ward identities in the BTGT formalism are satisfied to one loop by dimensional regularization just used. The $U(1)$ Ward identity (57) is satisfied by Eq. (88) since

$$
\begin{aligned}
\sum_{a}\left(i \Pi_{1}^{a b}+i \Pi_{2}^{a b}\right) \propto & \sum_{a} k_{\alpha}\left(H^{a}\right)^{\alpha \mu} P_{T \mu \nu} \frac{2}{\varepsilon} \ldots \\
& =0
\end{aligned}
$$

where we used Eq. (A1). That is why we were able to absorb the divergence using only gauge-invariant counterterms. Next, the BTGT Ward identity (71) is satisfied by Eq. (88) since

$$
\begin{gathered}
i \Pi_{1}^{a b}\left(K_{\beta}^{(\perp a)}(k)\right)+i \Pi_{2}^{a b}\left(K_{\beta}^{(\perp a)}(k)\right) \propto \sum_{j \neq a} k_{\lambda}\left(H^{j}\right)_{\alpha}^{\lambda}\left(H^{a}\right)^{\alpha \mu} \underset{\varepsilon}{2} \ldots \\
=0
\end{gathered}
$$

where we used Eq. (A3). Hence, dimensional regularization also preserves the two-point function BTGT Ward identity.

\section{Vertex correction}

In this subsection, we compute the vertex corrections shown in Fig. 3. Let us first consider the diagram in Fig. 3(a). We find with a $\theta^{c}$ insertion

$$
\begin{aligned}
i \Gamma_{c}^{(1)}= & \sum_{a, b} \int \frac{d^{4} l}{(2 \pi)^{4}} e\left(k_{1}+l\right) \star_{a} l\left(\frac{i}{l^{2}-m^{2}}\right) \\
& \times e(2 l+k) \star_{c} k\left(\frac{i}{(l+k)^{2}-m^{2}}\right)\left[-e(2 k+l) \star_{b} l\right] \\
& \times\left(\frac{-i}{l^{2}}\right) \delta^{a b} \frac{1}{l \star_{b} l} \\
= & -i k \star_{c}\left(i \Gamma^{\text {ordinary } 1}\right)
\end{aligned}
$$

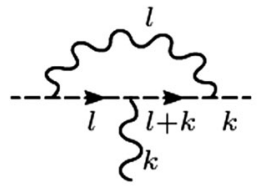

(a)

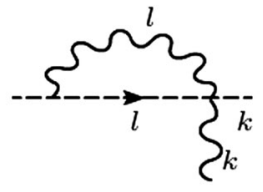

(b)

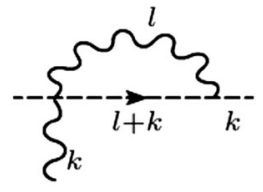

(c)
FIG. 3. One-loop cubic vertex corrections are shown in (a)-(c) when the scalar quartic self-coupling is zero at tree level. Note that we have set the incoming scalar momentum to zero to simplify the computation as is sometimes done [30]. 
where

$$
\begin{aligned}
i\left(\Gamma^{\text {ordinary } 1}\right)^{\kappa} & \equiv e^{3} \int \frac{d^{4} l}{(2 \pi)^{4}} \frac{(2 l+k)^{\kappa}}{l^{2}-m^{2}} \frac{(2 k+l)^{\lambda}}{(l+k)^{2}-m^{2}} \frac{l_{\lambda}}{l^{2}} \\
& =e^{3}\left[k^{\kappa} \frac{i}{(4 \pi)^{2}} \frac{2}{\varepsilon}+\cdots\right] .
\end{aligned}
$$

Next, the diagram Fig. 3(b) evaluates to

$$
\begin{aligned}
i \Gamma_{c}^{(2)}= & \sum_{a b} \int \frac{d^{4} l}{(2 \pi)^{4}} e(l) \star_{a} l\left(\frac{i}{l^{2}-m^{2}}\right)\left(-2 i e^{2}[-l] \star_{b} k \delta^{c b}\right) \\
& \times\left(\frac{-i}{l^{2}} \delta^{a b} \frac{1}{l \star_{b} l}\right) \\
= & 2 i e^{3} k_{\gamma}\left(H^{c}\right)^{\lambda \gamma} \int \frac{d^{4} l}{(2 \pi)^{4}} \frac{1}{l^{2}-m^{2}} \frac{l_{\lambda}}{l^{2}}
\end{aligned}
$$

which vanishes because of Lorentz symmetry and the fact that we have set the incoming scalar momentum to zero.

The most interesting diagram is Fig. 3(c),

$$
\begin{aligned}
i \Gamma_{c}^{(3)}= & \sum_{a b} \int \frac{d^{4} l}{(2 \pi)^{4}}\left(-2 i e^{2} k \star_{a} l \delta^{c a}\right) \frac{i}{(l+k)^{2}-m^{2}} \\
& \times\left(e[l+2 k] \star_{b}[-l]\right)\left(\frac{-i}{l^{2}} \delta^{a b} \frac{1}{l \star_{b} l}\right) \\
= & -i k_{\gamma}\left(H^{c}\right)^{\gamma \kappa}\left(I_{\xi_{3}}\right)_{\kappa}+i \underline{\Gamma}_{c}^{(3)}
\end{aligned}
$$

where

$$
\begin{aligned}
\left(I_{\xi_{3}}\right)_{\kappa} & \equiv-2 e^{3} \int \frac{d^{4} l}{(2 \pi)^{4}} \frac{1}{(l+k)^{2}-m^{2}} \frac{l_{\kappa}}{l^{2}} \\
& =e^{3} k_{\kappa}\left(\frac{i}{(4 \pi)^{2}} \frac{2}{\varepsilon}+\cdots\right)
\end{aligned}
$$

and

$i \underline{\Gamma}_{c}^{(3)} \equiv 4 i e^{3} k_{\alpha}\left(H^{c}\right)^{\alpha \psi} k_{\beta}\left(H^{c}\right)^{\beta \delta} \int \frac{d^{4} l}{(2 \pi)^{4}} \frac{l_{\psi} l_{\delta}}{(l+k)^{2}-m^{2}} \frac{1}{l^{2}} \frac{1}{l \star_{c} l}$,

which a priori looks different from the usual computation particularly because of the tensor structure in the denominator. By boosting to the diagonal frame of $H^{c}$, we can simplify this. Afterwards, we boost back to find

$$
i \underline{\Gamma}_{c}^{(3)}=4 i e^{3}\left(H^{c}\right)^{\mu \nu} k_{\mu} k_{\nu}\left(\frac{i}{(4 \pi)^{2}}\left[\frac{2}{\varepsilon}+\cdots\right]\right) .
$$

Combining this with Eq. (101), we find the third diagram contributes

$$
i \Gamma_{c}^{(3)}(k)=-3 e^{3} \frac{k \star_{c} k}{(4 \pi)^{2}}\left[\frac{2}{\varepsilon}+\cdots\right] .
$$

Combining the three vertex correction diagrams, we thus arrive at

$$
\begin{aligned}
i \Gamma_{c} & =i \sum_{n=1}^{3} \Gamma_{c}^{(n)} \\
& =-i k \star_{c}\left(i \Gamma^{\text {ordinary }}\right),
\end{aligned}
$$

where

$i\left(\Gamma^{\text {ordinary }}\right)^{\kappa} \equiv-i \frac{3 e^{3}}{8 \pi^{2} \varepsilon} k^{\kappa}+\left.k^{\kappa} \frac{i}{(4 \pi)^{2}} \frac{2}{\varepsilon} \xi e^{3}\right|_{\xi=1}+$ finite

$$
=-i \frac{e^{3}}{4 \pi^{2} \varepsilon} k^{\kappa}+\text { finite. }
$$

This leads to (for example, in the minimal subtraction renormalization scheme)

$$
Z_{1}=1+\frac{e^{2}}{4 \pi^{2} \varepsilon}
$$

With Eq. (108), we can also check that the three-point BTGT Ward identity (79) is preserved by the dimensional regularization. Before adding the counterterm, we have the regularized contribution

$$
\hat{G}_{a}^{(1,1,1)}\left(k_{1}^{\mu}, k_{2}^{\mu},-\left[K^{(\perp a)}(k)\right]^{\mu}\right) \ni-\left.k \star_{a} k \frac{e^{3}}{4 \pi^{2} \varepsilon}\right|_{k=-K^{(\perp a)}(k)}
$$

$$
=0
$$

satisfying the BTGT Ward identity. To check the $U(1)$ Ward identity, we still need the two-point function for the scalars.

\section{Scalar kinetic correction}

Let is now compute the one-loop scalar kinetic correction to check the Ward identity involving the three-point and two-point functions. Figure 4(a) gives 


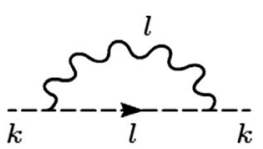

(a)

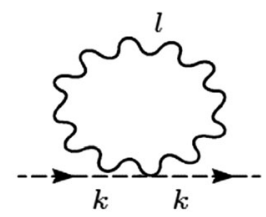

(b)
FIG. 4. One-loop scalar kinetic corrections are shown in (a)-(b) when the scalar quartic self-coupling is zero at tree level.

$$
\begin{aligned}
i \Pi_{(s 1)}= & \sum_{a b} \int \frac{d^{4} l}{(2 \pi)^{4}} e[2 k+l] \star_{a} l\left(\frac{i}{(k+l)^{2}-m^{2}}\right) \\
& \times e[2 k+l] \star_{b}[-l]\left(\frac{-i}{l^{2}} \delta^{a b} \frac{1}{l \star_{b} l}\right),
\end{aligned}
$$

where the novel tensor structure in the denominator can be handled by boosting to the $\left(H^{b}\right)^{\mu}{ }_{\nu}$ diagonal frame as in the vertex corrections. This results in

$$
i \Pi_{(s 1)}=-2 e^{2} k^{2} \frac{i}{(4 \pi)^{2}} \frac{2}{\varepsilon}+\cdots,
$$

which matches the usual computation results. The diagram of Fig. 4(b) gives

$$
\begin{aligned}
i \Pi_{(s 2)} & =-\sum_{a b} \int \frac{d^{4} l}{(2 \pi)^{4}} 2 i e^{2} l \star_{b} l \delta^{a b} \frac{-i}{l^{2}} \frac{1}{l \star_{b} l}+\cdots \\
& \propto \int \frac{d^{4} l}{(2 \pi)^{4}} \frac{1}{l^{2}}+\cdots,
\end{aligned}
$$

which has no novel tensor structure (as the numerator and the denominator cancel) and does not contribute to the $Z_{2}$ counterterm in dimensional regularization as usual.

Using Eq. (115) and the $i\left(Z_{2}-1\right) k^{2}$ counterterm, we thus find

$$
Z_{2}=1+\frac{e^{2}}{4 \pi^{2} \varepsilon}
$$

We explicitly see that $Z_{1}=Z_{2}$ in the minimal subtraction scheme that is the prediction of the $U(1)$ Ward identity.

Now, let us check that dimensional regularization satisfies the $U(1)$ Ward identity mixing the three-point function and the two-point function within the BTGT formalism. Using Eq. (108), we find the left-hand side of Eq. (62) before adding the counterterm is

$$
\sum_{c}\left(\frac{e^{3}}{4 \pi^{2} \varepsilon} k \star_{c} k+\cdots\right)=\frac{e^{3}}{4 \pi^{2} \varepsilon} k \cdot k+\cdots
$$

while we find the right-hand side from Eq. (115) to be

$$
\begin{aligned}
i e\left[\hat{G}^{(1,1,0)}(k,-k)-\hat{G}^{(1,1,0)}(0,0)\right] & =i e\left[-4 e^{2} k^{2} \frac{i}{(4 \pi)^{2} \varepsilon}\right] \\
& =k^{2} \frac{e^{3}}{4 \pi^{2} \varepsilon} .
\end{aligned}
$$

Hence, the $U(1)$ Ward identity is preserved by the dimensional regularization within the BTGT formalism as expected.

\section{E. Four-point function}

In this subsection, we would like to check the $U(1)$ Ward identity prediction $Z_{1}=Z_{4}$ for scalar QED. If we turn off the scalar quartic self-interaction, we only need to evaluate the diagram shown in Fig. 5,

$$
\begin{aligned}
i V_{a b}(A)= & \sum_{c e} \int \frac{d^{4} l}{(2 \pi)^{4}}\left(-2 i e^{2} k_{1} \star_{a} l \delta^{a c}\right)\left(\frac{i}{\left(l+k_{1}\right)^{2}-m^{2}}\right) \\
& \times\left(-2 i e^{2} k_{2} \star_{b}[-l] \delta^{b e}\right)\left(\frac{-i}{l^{2}} \delta^{c e} \frac{1}{l \star_{e} l}\right) .
\end{aligned}
$$

where $k_{1}$ is the incoming left external photon momentum and $k_{2}$ is the incoming right external photon momentum. Note that, unlike in the case of $A_{\mu}$ field theory, we cannot set $k_{1}=0$ to obtain the desired counterterm. To handle the BTGT specific tensor structure in the denominator, we can go to the $\left(H^{f}\right)_{\nu}^{\mu}$ diagonal basis as done before to evaluate this integral. We find

$$
i V_{a b}(A)=4 e^{4} \delta^{a b}\left(k_{1} \star_{b} k_{2}\right)\left(\frac{i}{(4 \pi)^{2}} \frac{2}{\varepsilon}+\text { finite }\right),
$$

where there is no sum over the repeated indices here. Adding this to the counterterm, the renormalization constant $Z_{4}$ can be extracted in the minimal subtraction scheme as

$$
Z_{4}=1+\frac{e^{2}}{4 \pi^{2} \varepsilon}
$$

matching the expected result

$$
Z_{4}=Z_{1}=Z_{2}
$$

of the $U(1)$ Ward identity.

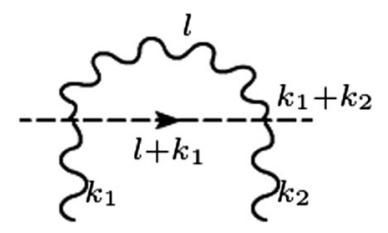

FIG. 5. Four-point function diagram that will contribute to the $Z_{4}$ at one loop if we turn off the quartic scalar self-coupling at tree level. 
Given that the $Z_{i}$ at one loop has the same result as in the ordinary formulation of scalar QED, we know that the $\beta$ function for this theory will be the same, i.e.,

$$
\frac{d \alpha}{d \ln \mu}=\frac{\alpha^{2}}{6 \pi},
$$

where $\alpha \equiv e^{2}(4 \pi)^{-1}$. Furthermore, this explicit computation of $Z_{4}$ was a nontrivial test of the (two $\theta$ )-(two scalar) coupling loop computation within the BTGT formalism.

\section{CONCLUSIONS}

In this work, we have investigated the Ward identities in the BTGT formalism associated with the BTGT symmetry and the $U(1)$ symmetry. As can be seen in Eq. (20), the BTGT symmetry current $\mathcal{B}_{a}^{\mu}$ can be classically interpreted as the basis tensor component decomposition of the $A^{\mu}$ equation of motion. Furthermore, the $U(1)$ current conservation and the conservation of a particular sum of the BTGT symmetry currents imply the same equation as the residual gauge symmetry current conservation as can be seen in Eq. (31). The $U(1)$ Ward identities for the two- and three-point functions in the BTGT formalism are displayed in Eqs. (48), (57), (61), and (62), while those for the BTGT Ward identities are displayed in Eqs. (69), (71), (77), and (79).

To check whether or not dimensional regularization is consistent with the BTGT symmetry Ward identity and the BTGT formalism in general, explicit one-loop renormalization of scalar QED was carried out. All two- and three-point Ward identities associated with BTGT symmetry and $U(1)$ are shown to be consistent with dimensional regularization. Novel dot products in the form of $A \star_{c} B$ appear both in the numerator and the denominator, but the renormalization constants in the minimal subtraction scheme are identical to the results from the standard computational formalism. It is clear from these explicit computations that the BTGT formalism is stable at one loop.

There are many future research directions for BTGT. It would be interesting to see if the non-Abelian gauge theories can be expressed in the BTGT formalism. This involves constructing a solution to the nonlinear constraint equation in a fashion similar to what was done for the Abelian theory. It would also be interesting to find practical applications for this theory in computing nonlocal correlators or in lattice gauge theory. This formalism should also be tested in the contexts of spontaneous symmetry breaking and curved spacetime. Since the $\theta^{a}$ field is very close to a Wilson line and since it is charged under a BTGT symmetry, the Abelian BTGT symmetry may be related to generalized global symmetries [31]. It would be worth investigating the precise connection. For physics beyond the standard model, it would be interesting to see if the basis tensor fields

$$
G_{\mu}^{\beta}(x)=\left[e^{i \theta^{a} H^{a}}\right]_{, \mu}^{\beta}
$$

can be embedded into a spontaneously broken theory since these fields are suggestive of a sigma model.

\section{ACKNOWLEDGMENTS}

This work was supported in part by the DOE through Grant No. DE-SC0017647.

\section{APPENDIX: USEFUL IDENTITIES}

The basis tensor matrices $\left(H^{a}\right)^{\mu}{ }_{\nu}$ were introduced in Ref. [20] to solve the nonlinear constraint equations. In the same sense in which $\gamma^{\mu}$ can be viewed to transform under Lorentz transformations, $\left(H^{a}\right)^{\mu}{ }_{\nu}$ can be thought of as an object transforming as a $(1,1)$ tensor under Lorentz transformations.

The basis tensor matrices have the following properties that are useful for Feynman diagram computations:

$$
\begin{gathered}
\sum_{a}\left(H^{a}\right)^{\mu}{ }_{\nu}=\delta^{\mu}{ }_{\nu} \\
\left(H^{a}\right)^{\mu \nu}=\left(H^{a}\right)^{\nu \mu} \\
\left(H^{a}\right)_{\nu}^{\mu}\left(H^{b}\right)^{\nu \lambda}=\delta^{a b}\left(H^{a}\right)^{\mu \lambda} \quad \text { no sum over } a .
\end{gathered}
$$

These matrices also serve as a kind of metric in BTGT characteristic dot products,

$$
A \star_{b} B \equiv A^{\mu} B^{\nu}\left(H^{b}\right)_{\mu \nu},
$$

where $A$ and $B$ are Lorentz 4-vector quantities.

Some of our other conventions used in this paper are as follows:

$$
\begin{gathered}
P_{T \mu \nu} \equiv \eta_{\mu \nu}-\frac{k_{\mu} k_{\nu}}{k^{2}} \\
P_{L \mu \nu} \equiv \frac{k_{\mu} k_{\nu}}{k^{2}} \\
\eta_{\mu \nu}=\operatorname{diagonal}(1,-1,-1,-1) .
\end{gathered}
$$

Finally, one should note that there is a typo in Eq. (36) of Ref. [20]. For Eq. (36) to be consistent with Eq. (39), we should define Eq. (36) to be

$$
\left(H^{a}\right)^{\mu}{ }_{\nu}=\psi_{(a)}^{\mu} \psi_{(a) \nu} \eta^{a a} \quad \text { no sum over } a,
$$

where

$$
\psi_{(a)}^{\mu} \equiv \Lambda_{a}^{\mu}
$$

is the Lorentz boost matrix that boosts away from the diagonal basis. 
[1] H. Weyl, A new extension of relativity theory, Ann. Phys. (Berlin) 364, 101 (1919).

[2] H. Weyl, Electron and gravitation. 1, Z. Phys. 56, 330 (1929) (in German).

[3] C.-N. Yang and R. L. Mills, Conservation of isotopic spin and isotopic gauge invariance, Phys. Rev. 96, 191 (1954).

[4] E. S. Abers and B. W. Lee, Gauge theories, Phys. Rep. 9, 1 (1973).

[5] C. Itzykson and J. B. Zuber, Quantum Field Theory, International Series In Pure and Applied Physics (McGraw-Hill, New York, 1980).

[6] A. M. Polyakov, Gauge fields, and strings, Contemp. Concepts Phys. 3, 1 (1987).

[7] G. 't Hooft, Under the spell of the gauge principle, Adv. Ser. Math. Phys. 19, 1 (1994).

[8] S. Weinberg, Modern Applications, The Quantum Theory of Fields (Cambridge University Press, Cambridge, England, 2013), Vol. 2.

[9] S. L. Glashow, Partial symmetries of weak interactions, Nucl. Phys. 22, 579 (1961).

[10] S. Weinberg, A Model of Leptons, Phys. Rev. Lett. 19, 1264 (1967).

[11] A. Salam, Weak and electromagnetic interactions, Conf. Proc. C680519, 367 (1968).

[12] D. J. Gross and F. Wilczek, Ultraviolet Behavior of Nonabelian Gauge Theories, Phys. Rev. Lett. 30, 1343 (1973).

[13] H. D. Politzer, Reliable Perturbative Results for Strong Interactions?, Phys. Rev. Lett. 30, 1346 (1973).

[14] P. Ramond, Journeys beyond the standard model, Front. Phys. 101, 1 (1999).

[15] P. Langacker, The Standard Model and Beyond, (CRC Press, Boca Raton, FL, 2010).

[16] G. Aad et al. (ATLAS Collaboration), Observation of a new particle in the search for the Standard Model Higgs boson with the ATLAS detector at the LHC, Phys. Lett. B 716, 1 (2012).
[17] S. Chatrchyan et al. (CMS Collaboration), Observation of a new boson at a mass of $125 \mathrm{GeV}$ with the CMS experiment at the LHC, Phys. Lett. B 716, 30 (2012).

[18] M. Nakahara, Geometry, Topology and Physics, (Taylor \& Francis, London, 2003).

[19] T. T. Wu and C. N. Yang, Concept of nonintegrable phase factors and global formulation of gauge fields, Phys. Rev. D 12, 3845 (1975).

[20] D. J.H. Chung and R. Lu, Basis tensor gauge theory: Reformulating gauge theories with basis tensor fields, Phys. Rev. D 94, 105016 (2016).

[21] K. G. Wilson, Confinement of quarks, Phys. Rev. D 10, 2445 (1974).

[22] R. Giles, The reconstruction of gauge potentials from Wilson loops, Phys. Rev. D 24, 2160 (1981).

[23] A. A. Migdal, Loop equations and $1 / \mathrm{N}$ expansion, Phys. Rep. 102, 199 (1983).

[24] J. Terning, Gauging nonlocal Lagrangians, Phys. Rev. D 44, 887 (1991).

[25] D. J. Gross, A. Hashimoto, and N. Itzhaki, Observables of noncommutative gauge theories, Adv. Theor. Math. Phys. 4, 893 (2000).

[26] A. Kapustin, Wilson-'t Hooft operators in four-dimensional gauge theories and S-duality, Phys. Rev. D 74, 025005 (2006).

[27] I. O. Cherednikov and N. G. Stefanis, Wilson lines and transverse-momentum dependent parton distribution functions: A renormalization-group analysis, Nucl. Phys. B802, 146 (2008).

[28] S. Mandelstam, Quantum electrodynamics without potentials, Ann. Phys. (Paris) 19, 1 (1962).

[29] J. C. Ward, An Identity in quantum electrodynamics, Phys. Rev. 78, 182 (1950).

[30] M. Srednicki, Quantum Field Theory (Cambridge University Press, Cambridge, England, 2007).

[31] D. Gaiotto, A. Kapustin, N. Seiberg, and B. Willett, Generalized global symmetries, J. High Energy Phys. 02 (2015) 172. 